\title{
Optimized Needle Shape Reconstruction Using Experimentally-Based Strain Sensors Positioning
}

\author{
Pierre-Loup Schaefer $^{1}$ - Grégory Chagnon ${ }^{1}$ - Alexandre Moreau-Gaudry ${ }^{2}$
}

Received: date / Accepted: date

\begin{abstract}
Needles are tools that are used daily during minimally invasive procedures. During the insertions needles may be affected by deformations which may threaten the success of the procedure. To tackle this problem, needles with embedded strain sensors have been developed and associated with navigation systems. The localization of the needle in the tissues is then obtained in real-time by reconstruction from the strain measurements, allowing the physician to optimize its gesture. As the number of strain sensors embedded is limited in number, their positions on the needle have a great impact on the accuracy of the shape reconstruction. The main contribution of this paper is a novel strain sensor positioning method to improve the reconstruction accuracy. A notable feature of our method is the use of experimental needle insertion data, which increases the relevancy of the resulting sensor optimal locations. To the best of author's knowledge no experimentally-based needle sensor positioning method has been presented yet. Reconstruction validations from clinical data show that the localization accuracy of the needle tip is improved by almost $40 \%$ with optimal locations compared to equidistant locations when reconstructing with two sensor triplets or more.
\end{abstract}

Keywords Instrumented needle Needle reconstruction Sensor locations $\cdot$ Shape estimation $\cdot$ Strain sensor

Number of words of the abstract: 186 words

\section{Pierre-Loup Schaefer}

E-mail: pierre-loup.schaefer@univ-grenoble-alpes.fr

Grégory Chagnon

E-mail: gregory.chagnon@univ-grenoble-alpes.fr

Alexandre Moreau-Gaudry

E-mail: alexandre.moreau-gaudry@univ-grenoble-alpes.fr

${ }^{1}$ Univ. Grenoble Alpes, CNRS, Grenoble INP, TIMC-IMAG, 38000 Grenoble, France

${ }^{2}$ Univ. Grenoble Alpes, CNRS, Grenoble INP, CHU Grenoble Alpes, TIMC-IMAG, 38000 Grenoble, France
Number of words of the manuscript: 4876 words

Number of figures: 7

Number of tables: 5

\section{Introduction}

Needles are tools daily used during minimally invasive procedures, such as in brachitherapy and biopsies [29]. These therapeutic gestures require to guide the needle to a target located in the patient body. To achieve this goal, physicians need to visualize the needle's position in real-time in the tissue. Different navigation systems have been thus developed to locate the needle in the tissue such as intraoperative systems using ultrasound or computed tomography. These systems have some drawbacks such as low precision or irradiations. To overcome these issues, some navigation systems use preoperative images of the patient associated with spatial registration of the needle to localize the needle in the patient tissue [41]. Most of these systems use the hypothesis that the needle remains straight during the insertion. Unfortunately the needle may bend during the insertion because of its interaction with the tissues. Experimental insertions in phantom models using 18 gauge $200 \mathrm{~mm}$ needles have shown that tip deflection can be as high as $2.8 \mathrm{~mm}$ for a 6 $\mathrm{cm}$ insertion [46] and $12 \mathrm{~mm}$ for a $10 \mathrm{~cm}$ insertion [32]. In this case, the hypothesis of a straight needle can have consequences such as attaining an undesired area of the tissues, leading to negative therapeutic results. Thus, in order to provide the deformed shape of the needle to the physicians using those systems, needle with embedded strain sensors have been developed. The goal is to increase the accuracy of the positioning of the needle in the tissues in order to improve the therapeutic results.

Instrumenting needle with strain sensors was first introduced by Park et al. [31] using Fiber Bragg gratings embed- 
ded on the needles to retrieve strain informations and use them to reconstruct the deformed shape. Since then, needle instrumented with strain sensors has been an intense topic of research with works by Henken et al. [16, 17], Abayazid et al. [1], Seifabadi et al. [42] or Roesthuis et al. [39, 40]. One of the most crucial aspect of the works is the accuracy of reconstruction of the needle deformed shape. As the technical limitations restricts the number of locations measures on the needle, the main problem consists in reconstructing a full deformed needle shape with only few strain measurements. Two aspects who play a crucial role in the reconstruction accuracy along the sensor sensibility are the needle shape reconstruction method and the locations of the sensors measures on the needle. Different reconstruction methods have been proposed for needles depending on the hypothesis made on the deformed needle shape during the insertion. The small displacement methods based on Euler-Bernoulli beam theory and presented in Park et al. [31], Abayazid et al. [1] and Seifabadi et al. will be used when the tip deflection of the needle is small whereas the other reconstruction methods proposed by Moon et al. [28], Roesthuis et al. [39] and Henken et al. [17] can be used otherwise. This problem of reconstruction, known as beam shape sensing, has also been investigated by using 3D beam model such as Todd $e t$ al. [45] in the general case or by Xu et al. [50] in the field of robotic.

The problem of sensor positioning has been adressed by Kim et al. [20] and Mahoney et al. [26] for continuum robots and by Park et al. [31] and Seifabadi et al. for needles. In Kim et al. the curvature sensors are placed to minimize the reconstruction error of the shape which is represented as a linear combination of spatial functions. The spatial functions are determined from actual robot configurations. Mahoney et al. [26] generalized this method to other sensor types by using an information theoretic viewpoint and demonstrated that shape reconstruction and sensor placement are coupled problems, involving that the sensor optimal locations are dependant of the shape reconstruction method. Concerning the best sensor locations on a needle, Park et al. [31] and Seifabadi et al. [42] have both proposed a method which is based on the minimization of the reconstruction error of a reference set of deformed needles. This reference set of needles is constituted of 2D shapes of needles built from a force model using Euler-Bernoulli beam theory. The best sensor locations computed with this method are thus restricted to small tip deflections and plane deformations only. Moreover, because they are built from force models, the representativeness of the reference needles is not guaranteed therefore compromising the relevancy of the sensor positions.

As in the works of Park et al. [31] and Seifabadi et al. [42], the sensor positioning method proposed in this paper is based on the minimization of the reconstruction error of a reference needle set. But on the contrary of these two existing methods, the reference needle set is not built from arbitrary deformed needles using a force model but is based on actual configurations as suggested by Kim et al. [20] and Mahoney et al. [26] and consists instead of real deformed needles coming from medical images of experimental needle insertions. The reference needles is thus composed of 3D deformed needles shapes obtained by segmentation and smoothing from CT scans of needle insertions which are then used in combination with the beam model to obtain simulated strain measures. Our sensor positioning method is not then limited to $2 \mathrm{D}$ or small deflection deformations. It is believed that this approach increases the representativeness of the reference needle set and therefore more valuable sensor location resulting in a higher gain of reconstruction accuracy. The validation of these results from reconstruction simulations with clinical images of needle insertions shows that the mean accuracy is $40 \%$ better with the resulting sensor locations than with equidistant locations when reconstructing with two sensor triplets or more. To the best of our knowledge, no experimentally-based needle sensor positioning method has been presented yet.

Section 2 presents the medical images of needle insertions used in this article. Section 3 consider deflection hypothesis and their consequences on the accuracy of the needle shape reconstruction. Section 4 introduces the needle shape reconstruction method and the strain sensor positioning method. Results of optimal sensor location results are presented and validated in Section 5. Finally, discussion and conclusion of our method are proposed in Section 6 and Section 7.

\section{Material}

This section presents the two sets of medical images of needle insertions used in this study.

\subsection{Experimental Needle Insertions}

The first dataset is composed of 54 CT scans of experimental needle insertions into pig shoulder carried out by Robert et al. [37]. The needle insertions were performed on a fresh pig shoulder (approximate size: $300 \mathrm{~mm} \times 200 \mathrm{~mm}$ $\times 100 \mathrm{~mm}$ ) prepared in a plastic tray. The needles used for the insertions were stainless steel 22 gauge $200 \mathrm{~mm}$ long needles, standardly used in interventional radiology, with an estimated Young's modulus of $200 \mathrm{GPa}$. The choice of porcine tissue was driven by its extensive use in needle insertions experiments where it gives good performance as a substitute for human tissue [18, 48, 19, 44]. As $\mathrm{Ng}$ et al. [30] point out, "the best replica closest to human tissue is 


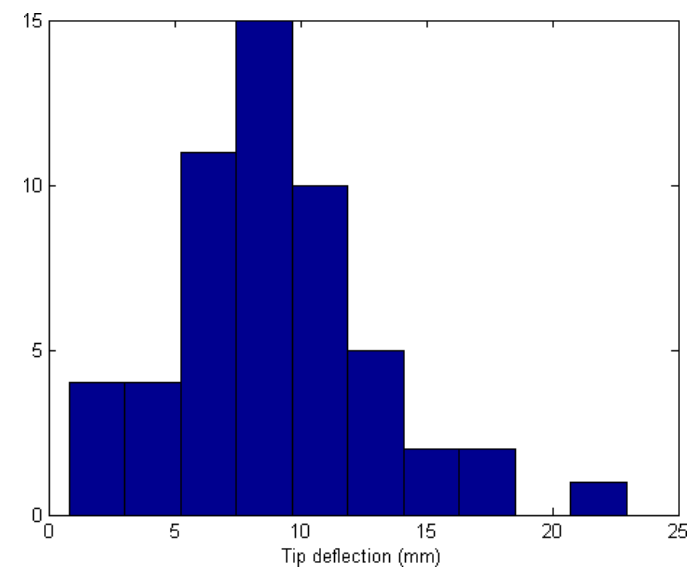

Fig. 1 Histogram of the tip deflection of the needles coming from the experimental needle insertions.

the use of porcine tissue from specific portion of the animals" ([30], p.187) and according to Brett et al. "models developed from porcine data will correlate with human specimens" ([7], p.341). Shoulder had been chosen for the presence of tendons, muscles and bones. The use of a such complex environment of insertion is driven by the necessity to provide the most complete range of needle deformations as possible [37]. A CT scan acquisition of the whole tissue volume was performed after each insertion. The insertions, as described in Robert et al. [37], have been performed using different characteristics every time (rotation, efforts, etc...), purposely obtaining the widest range of deformations possible to increase the representativity of the constituted set. The average tip deflection is $9 \mathrm{~mm}$ and the maximum tip deflection is $23 \mathrm{~mm}$. The histogram of the tip deflection of the needles is presented in Fig. 1.

\subsection{Clinical Needle Insertions}

The second dataset is composed of $10 \mathrm{CT}$ scans of a clinical trial performed at Grenoble-Alpes University Hospital, France. Different interventional radiology procedures were performed such as biopsies and injections. These CT scans were selected on the basis of needle characteristics criteria such as length, gauge and material. All the clinical CT scans used in this works thus features insertions using 22 gauges $200 \mathrm{~mm}$ stainless steel needles. On the opposite of the previous CT scans set presented in Section 2.1, the characteristics of the insertions of the needles are unknown. This clinical trial received the authorization of the ANSM, the French Agency for the Safety of Health Products, and the Comite de Protection des Personnes Sud Est V, the regional authority for human research protocols (ClinicalTrials.gov Identifier: NCT00828893). With an average of $5 \mathrm{~mm}$ and a maximum

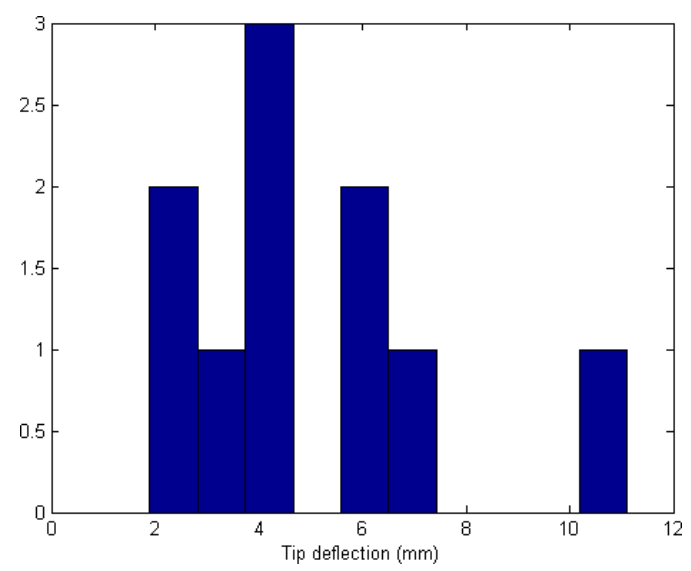

Fig. 2 Histogram of the tip deflection of the needles coming from the clinical needle insertions.

of $11 \mathrm{~mm}$ the deflections of the needle tip are smaller than in the case of the experimental needle insertions.

\section{Small deflection vs large deflection hypothesis: consequences on the needle shape reconstruction accuracy}

The main contributions of this paper is a needle shape reconstruction method covering 3D deformations and large deflections and a strain sensor positioning method based on experimental data. The needle deflection is the distance at any of its point from its reference straight configuration when subjected to deformation. The deflections are usually considered large when the deflection is higher than $10 \%$ of the beam length and are considered small otherwise. Large deflections are not always taken into account when reconstructing deformed needle shapes such as in Park et al. [31], Abayazid et al. [1] and Seifabadi et al. [42]. The impact of the small displacement hypothesis on the beam shape reconstruction will be evaluated here by considering the distance between the tips of needle shapes reconstructed with smalldisplacement and large-displacement hypothesis. For simplification purposes only cases of 2D bending deformations will be considered. An initial straight beam of length $L$ will be considered with 2D coordinates being noted $(x, y(x))$. In the reference configuration the beam is undeformed and thus parallel to the $\mathrm{x}$-axis: $y(x)=0$. The expression of the curvature of the beam in the deformed configuration is noted $\kappa$ and is defined as follows:

$\frac{y^{\prime \prime}(x)}{\left(1+y^{\prime 2}(x)\right)^{3 / 2}}=\kappa(x)$

which gives by integration:

$\frac{y^{\prime}(x)}{\sqrt{1+y^{\prime 2}(x)}}=\int_{0}^{x} \kappa(u) d u$ 
Table 1 Tip error of needle shape reconstructions with small displacement beam theory.

\begin{tabular}{|c|c|c|c|c|c|c|}
\hline \multicolumn{3}{|c|}{ Needle characteristics } & \multicolumn{3}{c|}{ Deformed needle shape tips comparison } \\
\hline$L(\mathrm{~mm})$ & $Y(L)(\mathrm{mm})$ & $Y(L) / L(\%)$ & $\Delta_{x}(L)(\mathrm{mm})$ & $\Delta_{y}(L)(\mathrm{mm})$ & $d(L)(\mathrm{mm})$ & $d(L) / Y(L)(\%)$ \\
\hline 100 & 12 & $12 \%$ & 0.87 & 0.03 & 0.87 & $8 \%$ \\
\hline 200 & 11 & $6 \%$ & 0.36 & 0.01 & 0.36 & $4 \%$ \\
\hline 200 & 23 & $12 \%$ & 1.59 & 0.04 & 1.61 & $7 \%$ \\
\hline
\end{tabular}

The beam coordinates $(x, y(x))$ are parameterized by the arclength $s$ and noted $(x(s), y(x(s)))$. As only bending is considered, the beam length is supposed to remain constant during the deformation and the abscissa $x(s)$ of the beam at the arclength value $s$ is then:

$x(s)=x_{0} \in \mathbb{R} / s=\int_{0}^{x_{0}} \sqrt{1+y^{\prime}(x)^{2}} d x$

Using (2) and (3) we then have the following expression of the coordinate $y(x(s))$ of the beam [27]:

$y(x(s))=\int_{0}^{x(s)} \frac{\int_{0}^{v} \kappa(u) d u}{\sqrt{1-\left(\int_{0}^{v} \kappa(u) d u\right)^{2}}} d v$

The coordinates of the beam given by the linear EulerBernoulli beam theory using the small displacement hypothesis will be noted $(\tilde{x}, \tilde{y}(\tilde{x}))$. Under the small displacement hypothesis the value of the term $y^{\prime 2}$ in (1) is neglected and the expression of the curvature is then:

$\tilde{y}^{\prime \prime}(\tilde{x})=\kappa(\tilde{x})$

The beam coordinates $(\tilde{x}, \tilde{y}(\tilde{x}))$ are parameterized by the arclength $s$ and noted $(\tilde{x}(s), \tilde{y}(\tilde{x}(s)))$. Under the hypothesis of small displacement the coordinate $\tilde{x}(s)$ of the beam is:

$\tilde{x}(s)=s$

Using (5) and (6) we then have the following expression of the coordinate $\tilde{y}(\tilde{x}(s))$ of the beam:

$\tilde{y}(\tilde{x}(s))=\int_{0}^{s} \int_{0}^{v} \kappa(u) d u d v$

Consequently, the linear Euler-Bernoulli beam model with small displacement include one approximation for each of the coordinates. Firstly, as shown in (6), the expression of $\tilde{x}(s)$ remains constant during the deformation and thus does not take into account the deformation of the beam. Secondly, as shown in (5) and then in (7) the curvature is approximated which gives a simplified expression of the coordinate $\tilde{y}(s)$.

The distance $d$ at arclength $s$ between the beam shapes $(x(s), y(x(s)))$ and $(\tilde{x}(s), \tilde{y}(\tilde{x}(s)))$ is:

$d(s)=\sqrt{\Delta_{x}^{2}(s)+\Delta_{y}^{2}(s)}$

with:

$\Delta_{x}(s)=x(s)-\tilde{x}(s)$

$\Delta_{y}(s)=y(x(s))-\tilde{y}(\tilde{x}(s))$
The expression of the distance $d$ between the two reconstructed shapes depends obviously of the needle curvature $\kappa$. In order to obtain a realistic evaluation of what the maximum impact of the small displacement hypothesis can be on the needle shape reconstruction, the distance $d$ will be evaluated with curvature functions obtained from real deformed needles characteristics. Two large deflections and one small deflection needle deformations have thus been used to this end: the $12 \mathrm{~mm}$ deflection of a $100 \mathrm{~mm}$ needle presented in [32], the $23 \mathrm{~mm}$ deflection of a $200 \mathrm{~mm}$ needle coming from experimental needle insertions presented in Section 2.1 and the $11 \mathrm{~mm}$ deflection of a $200 \mathrm{~mm}$ needle coming from clinical neede insertions presented in Section 2.2. This characteristics of length and deflection are then used with 2D linear beam theory to obtain plausible curvatures of bending beams satisfying those characteristics. The distance between the two reconstructed end shapes $d(L)$ constitute the tip error of reconstruction and is presented for each of the deformations in Table 1. According to the results, the relative tip errors $d(L)$ appear to be mainly influenced by the coefficient $\Delta_{x}(L)$ and to be higher for large displacement deformations (7\% and $8 \%$ ) than for small displacement deformations (4\%). This means that the assumption made in (6) concerning the abscissa of the beam is the main factor of inaccuracy of Euler-Bernoulli beam theory for reconstructing needles subjected to simple bending and that the higher the tip deflection is, the higher the relative tip error of reconstruction will be. As results show, taking into account large displacement thus helps to improve the reconstruction accuracy in both cases of small and large deflections. The goal of this article being to improve the needle shape reconstruction accuracy by positioning strain sensors optimally, using large displacement beam theory will first of all improve the accuracy of the method of reconstruction of the needle shape.

The estimation of optimal sensor locations from experimental needle data requires strain computations of deformed needle shapes, hence the need to adopt a beam model including the material configuration of the beam. One of the most convenient way to handle material configuration was proposed by Cosserat [9] and consists in attaching a frame to each particle in the continuum. This parameterization was used by Reissner [35] to develop a 1D continuum large displacement 3D beam theory extending previous works of Kirchhoff [21] and Love [24]. The Cosserat 1D continuum parameterization associated with the large dis- 
placement modeling makes the Reissner beam theory perfectly designed to handle needle structures undergoing large deflections which might occur during needle insertions. The sensor positioning method proposed in this paper uses the frame parametrization of the beam to formulate a differential system expressing the deformation of the material frame of the needle in terms of strain configuration. This system is one of novelty of this work and the key element of both reconstruction process and optimal sensor positioning. In fact, its resolution provides a comprehensive shape reconstruction as an iterative method to obtain the shape of the needle from the strain measurements of the sensors. Conversely, associated with curve framing the system is used in the sensor positioning method to obtain the material frame of a needle from a given needle shape and simulate the measurements of the strain sensors.

\section{Methods}

\subsection{D Needle Shape Reconstruction for Large Deflections}

This section addresses the problem of reconstructing the shape of a needle using the data from its embedded strain sensors. First of all, the strain measures are acquired from the strain sensors and processed to retrieve the curvature and the bending angle, two geometrical functions characterizing the $3 \mathrm{D}$ deformation. The beam theory is then used to build a linear matrix differential system accounting of the needle deformation in terms of those two functions. This system is solved using a method of resolution for differential equation on a Lie algebra. Finally, an iterative scheme of reconstruction for the needle shape is then presented.

\subsubsection{Needle Curvature and Bending Angle}

In most articles dealing with needles with embedded strain sensors located at the surface of the needle, the sensors are oriented parallel to the needle to measure the axial strain and placed on needle cross sections by groups of three with a 120 degrees angle between them [39, 1, 31, 16, 42], as shown in Fig. 3. This orientation and distribution will be used in the rest of this paper for each cross sections where sensors are placed and will be called sensor triplets.

The deformation of a circular beam is presented in Fig. 4. The beam radius is noted $r_{0}$ and the cross section is defined by the intersection of the section plane $\mathscr{P}_{s}$ with the beam. The sensor triplet located on the cross section is composed of strain sensors $S_{1}, S_{2}$ and $S_{3}$ whose respective local strain measures are $\varepsilon_{1}, \varepsilon_{2}$ and $\varepsilon_{3}$. The intersection between the section plane $\mathscr{P}_{s}$ and the beam neutral axis is denoted $O$. The curvature of the beam neutral axis at point $O$ is denoted $\kappa$. The beam deformation is characterized by the plane of bending $\mathscr{P}_{b}$ which contains $C$, the center of the osculating

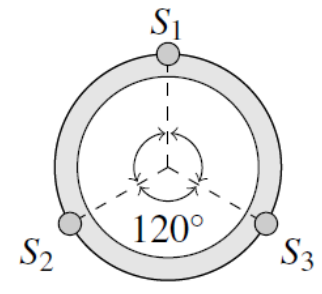

Fig. 3 Sensor triplets composed of strain sensors $S_{1}, S_{2}$, $S_{3}$ placed on a needle cross section with a 120 degrees angle between them.

circle to the beam neutral axis at point $O$. The points of cross section with maximum and minimum strains are denoted respectively $\varepsilon_{\max }$ and $-\varepsilon_{\max }$. The angle between the sensor $S_{1}$ and the point $\varepsilon_{\text {max }}$ is called the bending angle and is denoted $\theta$. For linear elastic deformations, it is possible to express the local strain measures $\varepsilon_{i}$ in terms of curvature $\kappa$, bending angle $\theta$ and bias due to other deformations than bending $\delta$ $[39,1,16]$ :

$$
\left\{\begin{array}{l}
\varepsilon_{1}=r_{0} \kappa \cos (\theta)+\delta \\
\varepsilon_{2}=r_{0} \kappa \cos \left(\theta-\frac{2 \pi}{3}\right)+\delta \\
\varepsilon_{3}=r_{0} \kappa \cos \left(\theta-\frac{4 \pi}{3}\right)+\delta
\end{array}\right.
$$

By solving the set of equations (11) the parameters $\kappa, \theta$ and $\delta$ are determined:

$$
\kappa=\frac{1}{3 r_{0}} \sqrt{2\left(\left(\varepsilon_{1}-\varepsilon_{2}\right)^{2}+\left(\varepsilon_{2}-\varepsilon_{3}\right)^{2}+\left(\varepsilon_{1}-\varepsilon_{3}\right)^{2}\right)}
$$

$$
\begin{aligned}
\cos (\theta) & =\frac{2 \varepsilon_{1}-\varepsilon_{2}-\varepsilon_{3}}{\sqrt{2\left(\left(\varepsilon_{1}-\varepsilon_{2}\right)^{2}+\left(\varepsilon_{2}-\varepsilon_{3}\right)^{2}+\left(\varepsilon_{1}-\varepsilon_{3}\right)^{2}\right)}} \\
\sin (\theta) & =\frac{\sqrt{3}\left(\varepsilon_{2}-\varepsilon_{3}\right)}{\sqrt{2\left(\left(\varepsilon_{1}-\varepsilon_{2}\right)^{2}+\left(\varepsilon_{2}-\varepsilon_{3}\right)^{2}+\left(\varepsilon_{1}-\varepsilon_{3}\right)^{2}\right)}} \\
\delta & =\frac{\varepsilon_{1}+\varepsilon_{2}+\varepsilon_{3}}{3}
\end{aligned}
$$

Considering a needle instrumented with $n$ strain sensor triplets, the curvature and the bending angle values at the location $s_{i}$ of the $i^{\text {th }}$ triplet are noted respectively $\kappa_{i}$ and $\theta_{i}$. The estimate of curvature $\kappa_{e s t}$ and the estimate of bending angle $\theta_{\text {est }}$ can be obtained by interpolations of the sets $\left(\kappa_{i}\right)_{i=1, . ., n}$ and $\left(\theta_{i}\right)_{i=1, \ldots, n}$ over $[0, L]$ with the following boundary counditions:

$\begin{aligned} \kappa_{\text {est }}(L) & =0 \\ \theta_{\text {est }}(0) & =0\end{aligned}$ 


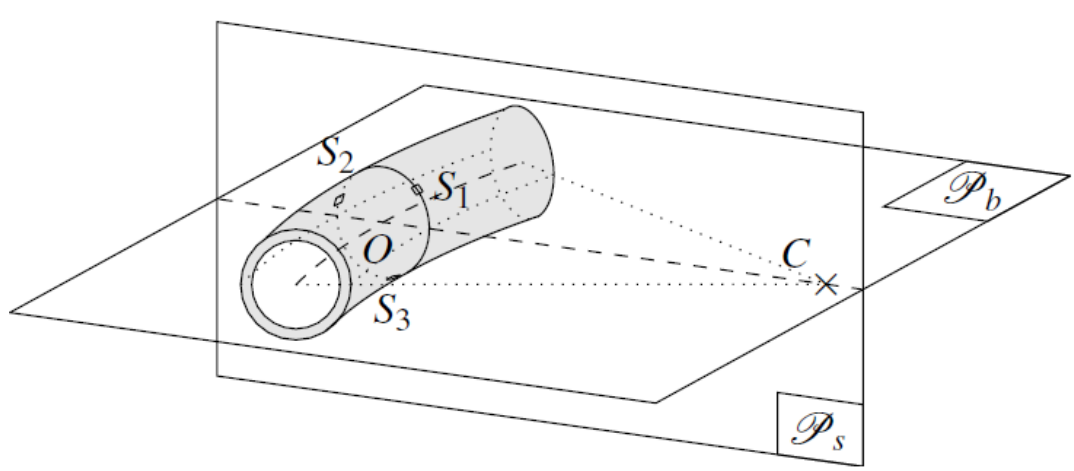

(a)

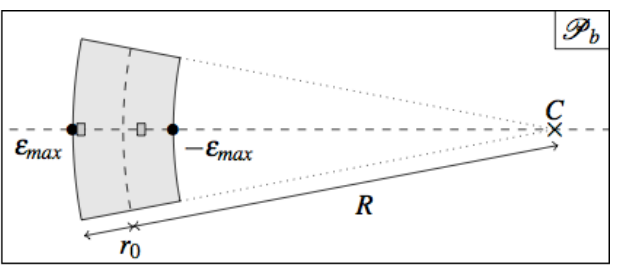

(b)

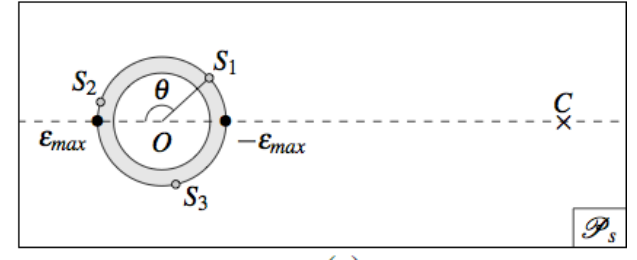

(c)

Fig. 4 (a) Deformation of a beam element. The section plane is denoted $\mathscr{P}_{s}$ and contains the sensor triplets $\left(S_{1}, S_{2}, S_{3}\right)$. The plane of bending is denoted $\mathscr{P}_{b}$ and contains $C$ the center of the osculating circle to the beam neutral axis at point $O$.

(b) Diagram of the plane of bending $\mathscr{P}_{b}$. The radius of curvature is denoted $R$. The points of cross section with maximum and minimum strains are denoted respectively $\varepsilon_{\max }$ and $-\varepsilon_{\max }$.

(c) Diagram of the section plane $\mathscr{P}_{s}$. The angle $\theta$ is called the bending angle and is defined as the angle between $S_{1}$ and $\varepsilon_{\max }$.

Condition from (16) reflects that curvature of the needle is null at the distal extremity due to the absence of bending moment there whereas condition from (17) is used to give an initial value of bending angle. The method of interpolation used is linear interpolation with the following initial conditions:

$\kappa_{\text {est }}(0)=\kappa_{1}$

$\theta_{\text {est }}(L)=\theta_{n}$

\subsubsection{Needle Deformation Model}

The needle is modeled at its initial state by a cylindrical straight beam of radius $r_{0}$ and length $L$. The needle being composed of isotropic material and its section being symmetric, the set of the centers of all the sections of the beam composes the neutral axis. Needle deformations due to shearing, tension and compression are neglected during insertions into tissue. The position of the beam neutral axis at length $s$ is denoted $\mathbf{r}(s)$ in the reference configuration and $\mathbf{R}(s)$ in the current configuration. The convected coordinates of the beam, which are embedded in the material and deform along with the material [34], are defined in the reference configuration by the orthonormal frame $\left(\mathbf{t}, \mathbf{n}_{1}, \mathbf{n}_{2}\right)$ with $\mathbf{t}$ tangent to the neutral axis $\mathbf{r}$ :

$\mathbf{t}=\mathbf{r}^{\prime}$

and such that:

$\mathbf{t}=\mathbf{e}_{1}, \quad \mathbf{n}_{1}=\mathbf{e}_{2}, \quad \mathbf{n}_{2}=\mathbf{e}_{3}$ with $\left(\mathbf{e}_{\mathbf{1}}, \mathbf{e}_{2}, \mathbf{e}_{3}\right)$ being the canonical basis of $\mathbb{R}^{3}$ as illustrated in Fig. 5a. The sensor triplets are placed on beam sections such that vectors $\mathbf{e}_{2}$ are directed at sensors $S_{1}$. The convected coordinates are defined in the current configuration by the orthonormal frame $\left(\mathbf{T}, \mathbf{N}_{\mathbf{1}}, \mathbf{N}_{\mathbf{2}}\right)$ as shown in Fig. 5b. As a consequence of (20) we then have:

$$
\mathbf{T}=\mathbf{R}^{\prime}
$$

The differentiation of $\left(\mathbf{T}, \mathbf{N}_{\mathbf{1}}, \mathbf{N}_{\mathbf{2}}\right)$ gives the following linear differential system [35]:

$$
\left[\begin{array}{c}
\mathbf{T} \\
\mathbf{N}_{\mathbf{1}} \\
\mathbf{N}_{2}
\end{array}\right]^{\prime}=\Omega\left[\begin{array}{c}
\mathbf{T} \\
\mathbf{N}_{\mathbf{1}} \\
\mathbf{N}_{\mathbf{2}}
\end{array}\right]
$$

with

$\Omega=\left[\begin{array}{ccc}0 & \varkappa_{1} & \varkappa_{2} \\ -\varkappa_{1} & 0 & \varkappa_{t} \\ -\varkappa_{2} & -\varkappa_{t} & 0\end{array}\right]$

The components of $\Omega$ are the torsion factor $\varkappa_{t}$ and the bending factors $\varkappa_{1}$ and $\varkappa_{2}$. During the insertion the mechanical torsion of the needle can be ignored because of the geometry of the needle, its mechanical properties and its interactions with the tissues, thus $[1,31]$ :

$$
\varkappa_{t}=0
$$

The components of $\Omega$ can be expressed in terms of curvature $\kappa$ and bending angle $\theta$ using the Serret-Frenet frame $(\mathbf{T}, \mathbf{N}, \mathbf{B})$ of the curve $\mathbf{R}$. The vector $\mathbf{T}$ is the tangent vector and is shared by the Frenet-Serret frame and the convected coordinates. The vector $\mathbf{N}$ is the normal vector and is 


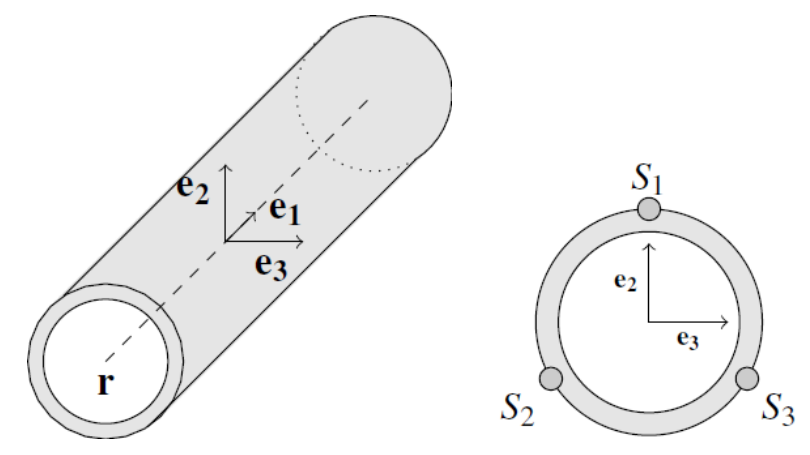

(a)
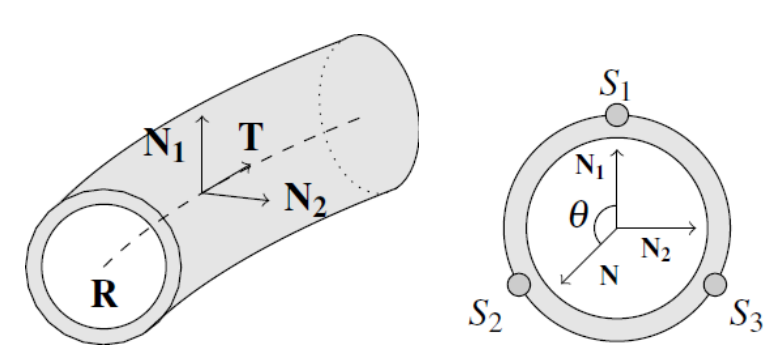

(b)

Fig. 5 (a) Needle convected coordinates before deformation $\left(\mathbf{e}_{\mathbf{1}}, \mathbf{e}_{\mathbf{2}}, \mathbf{e}_{\mathbf{3}}\right)$. The sensor triplet $\left(S_{1}, S_{2}, S_{3}\right)$ belongs to the plane $\left(\mathbf{e}_{2}, \mathbf{e}_{3}\right)$.

(b) Needle convected coordinates after deformation $\left(\mathbf{T}, \mathbf{N}_{\mathbf{1}}, \mathbf{N}_{\mathbf{2}}\right)$. The sensor triplet $\left(S_{1}, S_{2}, S_{3}\right)$ belongs to the plane $\left(\mathbf{N}_{\mathbf{1}}, \mathbf{N}_{\mathbf{2}}\right)$. The normal vector $\mathbf{N}$ is included in the plane of bending, directed towards the center of the osculating plane and is obtained with the rotation of $\mathbf{N}_{\mathbf{1}}$ by an angle $\theta$.

directed towards the center of the osculating circle and the vector $\mathbf{B}$ is the binomial vector. The derivative of $(\mathbf{T}, \mathbf{N}, \mathbf{B})$ can be expressed with the curvature $\kappa$ and the mathematical torsion $\tau$ :

$$
\left[\begin{array}{l}
\mathbf{T} \\
\mathbf{N} \\
\mathbf{B}
\end{array}\right]^{\prime}=\left[\begin{array}{ccc}
0 & \kappa & 0 \\
-\kappa & 0 & \tau \\
0 & -\tau & 0
\end{array}\right]\left[\begin{array}{l}
\mathbf{T} \\
\mathbf{N} \\
\mathbf{B}
\end{array}\right]
$$

Because $\mathbf{T}$ is a shared vector of both orthonormal frames, the convected coordinates $\left(\mathbf{T}, \mathbf{N}_{\mathbf{1}}, \mathbf{N}_{\mathbf{2}}\right)$ can be obtained from the Serret-Frenet frame $(\mathbf{T}, \mathbf{N}, \mathbf{B})$ by a rotation of axis $\mathbf{T}$ [12]. Thus the rotation angle transforms the vector $\mathbf{N}$ to the vector $\mathbf{N}_{\mathbf{1}}$ and the vector $\mathbf{B}$ to the vector $\mathbf{N}_{\mathbf{2}}$. As $\mathbf{N}$ is included in the plane of bending and $\mathbf{N}_{\mathbf{1}}$ is directed towards the sensor $S_{1}$ (Fig. 5b) the angle of rotation between the two frames is the previously defined bending angle $\theta$. We then have the following relation:

$$
\left[\begin{array}{c}
\mathbf{T} \\
\mathbf{N}_{\mathbf{1}} \\
\mathbf{N}_{\mathbf{2}}
\end{array}\right]=\left[\begin{array}{ccc}
1 & 0 & 0 \\
0 & \cos \theta & \sin \theta \\
0 & -\sin \theta & \cos \theta
\end{array}\right]\left[\begin{array}{l}
\mathbf{T} \\
\mathbf{N} \\
\mathbf{B}
\end{array}\right]
$$

The differentiation of (27) associated with (26) leads to the following expression:

$$
\left[\begin{array}{c}
\mathbf{T} \\
\mathbf{N}_{\mathbf{1}} \\
\mathbf{N}_{\mathbf{2}}
\end{array}\right]^{\prime}=\left[\begin{array}{ccc}
0 & \kappa & 0 \\
-\kappa \cos \theta & -\left(\theta^{\prime}+\tau\right) \sin \theta & \left(\theta^{\prime}+\tau\right) \cos \theta \\
\kappa \sin \theta & -\left(\theta^{\prime}+\tau\right) \cos \theta & \left(\theta^{\prime}+\tau\right) \sin \theta
\end{array}\right]\left[\begin{array}{l}
\mathbf{T} \\
\mathbf{N} \\
\mathbf{B}
\end{array}\right]
$$

Finally, using in (28) the expression of $(\mathbf{T}, \mathbf{N}, \mathbf{B})$ in terms of $\left(\mathbf{T}, \mathbf{N}_{\mathbf{1}}, \mathbf{N}_{\mathbf{2}}\right)$ obtained from (27) then gives the differential system defined in (23):

$$
\left[\begin{array}{c}
\mathbf{T} \\
\mathbf{N}_{\mathbf{1}} \\
\mathbf{N}_{\mathbf{2}}
\end{array}\right]^{\prime}=\left[\begin{array}{ccc}
0 & \kappa \cos \theta & -\kappa \sin \theta \\
-\kappa \cos \theta & 0 & \left(\theta^{\prime}+\tau\right) \\
\kappa \sin \theta & -\left(\theta^{\prime}+\tau\right) & 0
\end{array}\right]\left[\begin{array}{c}
\mathbf{T} \\
\mathbf{N}_{\mathbf{1}} \\
\mathbf{N}_{\mathbf{2}}
\end{array}\right]
$$

By taking the value of the torsion factor $\varkappa_{t}$ from (25) the expression of $\Omega$ is:

$\Omega=\kappa\left[\begin{array}{ccc}0 & \cos \theta & -\sin \theta \\ -\cos \theta & 0 & 0 \\ \sin \theta & 0 & 0\end{array}\right]$

The differential system (23) governing the evolution of the convected coordinates $\left(\mathbf{T}, \mathbf{N}_{\mathbf{1}}, \mathbf{N}_{\mathbf{2}}\right)$ can now be resolved.

\subsubsection{Iterative Needle Shape Reconstruction}

Equation (23) can be rewritten using the more practical form of a matrix differential equation:

$\mathbf{Y}^{\prime}=\Omega \mathbf{Y}, \quad \mathbf{Y}(0)=\mathbf{Y}_{0}$

with $\mathbf{Y}$ the matrix containing the coordinates of $\left(\mathbf{T}, \mathbf{N}_{\mathbf{1}}, \mathbf{N}_{\mathbf{2}}\right)$. The initial condition $\mathbf{Y}_{0}$ contains the coordinates of $\left(\mathbf{T}, \mathbf{N}_{\mathbf{1}}, \mathbf{N}_{\mathbf{2}}\right)$ at the proximal extremity of the needle.

Equation (31) is a differential equation on the matrix Lie group $S O(3)$, the $3 \mathrm{D}$ rotation group, ensuring the existence and uniqueness of a solution, which belongs to $S O(3)$ [13]. It will be solved using the structure preserving method Local Coordinates Approach [13] giving an approximate solution belonging to $S O(3)$.

The set $\left(s_{i}\right)_{i=0, N}$ is the spatial discretization of the length of the beam, with $s_{0}=0, s_{N}=L$ and $s_{n+1}=s_{n}+h, h=$ $L /(n-1)$ being the step of resolution. The method is presented for a step from $s_{n}$ to $s_{n+1}$. The approximation of $\mathbf{Y}\left(s_{n}\right)$ and $\mathbf{Y}\left(s_{n+1}\right)$ are denoted $\mathbf{Y}_{n}$ and $\mathbf{Y}_{n+1}$. The iterative step of the method is based on the Magnus expansion [25] which gives an exponential representation of the solution of first order matrix differential equations such as (31). Truncation of the Magnus expansion and approximation by midpoint rule gives the following iteration to obtain $\mathbf{Y}_{n+1}$ from $\mathbf{Y}_{n}$ which is a method of order 2 [13]:

$\mathbf{Y}_{n+1}=\exp \left(h \Omega_{e s t}\left(s_{n}+\frac{h}{2}\right)\right) \mathbf{Y}_{n}$ 
with $\Omega_{\text {est }}$ the estimate of $\Omega$ from (30) using the estimates $\kappa_{\text {est }}$ and $\theta_{\text {est }}$ as functions $\kappa$ and $\theta$. Equation (32) contains a matrix exponential. Using the formula for exponential mapping in $S O(3)$ gives the following expression [43]:

$\exp \left(h \Omega_{e s t}\left(s_{n}+\frac{h}{2}\right)\right)=\left[\begin{array}{ccc}c_{1} & s_{1} c_{2} & -s_{1} s_{2} \\ -s_{1} c_{2} & c_{1}+\alpha s_{2}^{2} & \alpha c_{2} s_{2} \\ s_{1} s_{2} & \alpha c_{2} s_{2} & c_{1}+\alpha c_{2}^{2}\end{array}\right]$

with:

$c_{1}=\cos \left(h \kappa_{\text {est }}\left(s_{n}+\frac{h}{2}\right)\right)$

$s_{1}=\sin \left(h \kappa_{e s t}\left(s_{n}+\frac{h}{2}\right)\right)$

$c_{2}=\cos \left(\theta_{\text {est }}\left(s_{n}+\frac{h}{2}\right)\right)$

$s_{2}=\sin \left(\theta_{\text {est }}\left(s_{n}+\frac{h}{2}\right)\right)$

$\alpha=1-h \kappa_{e s t}\left(s_{n}+\frac{h}{2}\right)$

Using relation (32) iteratively on the set $\left(s_{i}\right)_{i=1, . ., n}$ gives $\left(\mathbf{Y}_{i}\right)_{i=1, \ldots, n}$ containing the coordinates of $\mathbf{T}_{i}$ the approximation of $\mathbf{T}\left(s_{i}\right)$. We then have the set of the tangent approximations $\left(\mathbf{T}_{i}\right)_{i=1, . ., n}$. By using (22) we define $\mathbf{R}_{i}$, the approximation of $\mathbf{R}\left(s_{i}\right)$, using the recurrence relation:

$$
\begin{aligned}
\mathbf{R}_{0} & =\mathbf{R}\left(s_{0}\right) \\
\mathbf{R}_{i+1} & =\mathbf{R}_{i}+h \mathbf{T}_{i}
\end{aligned}
$$

Finally the set $\left(\mathbf{R}_{i}\right)_{i=1, \ldots, n}$ is the three-dimensional reconstruction of the shape of the deformed needle.

\subsection{Needle Sensor Positioning}

The problem of sensor positioning involves finding the sensor triplet locations on a needle which provide the best reconstruction accuracy. Such locations are called optimal sensor locations [42]. The reconstruction accuracy of a needle is evaluated by an error measure between its original and reconstructed shape. Thus, the optimal sensor locations are the locations that minimize in average this error measure [20]. In practice, Park et al.[31] and Seifabadi et al.[42] established the optimal sensor locations as the locations that minimize the mean of the error measure for some reference needles. Therefore, for given sensor locations, the reconstructed shape associated to a reference needle is obtained by needle shape reconstruction with the strain inputs being the strains of the reference needle at the sensor locations. In their works, Park et al. [31] and Seifabadi et al. [42] built the reference needles using Euler-Bernoulli beam theory to reconstruct needle shapes from a $2 \mathrm{D}$ force model. Thus, the deformations of their reference needles are restricted to 2D small deflections.

The sensor positioning method proposed in this paper is based on a novel approach using experimental needle insertions data to build the reference needle set. Figure 1 shows that the deflection of some needle of the experimental data can't be qualified as large but still have high values, comprised between $5 \%$ and $10 \%$ of the needle length. According to Section 1, the processing of such needles thus justifies the use of large displacement beam theory as the approximation using a small displacement beam theory would degrade the quality of treatment, especially on the abscissa parameterization. By using the deformation model presented in Section 4.1.2 it is then possible to take complex deformations into account, such as 3D deformations or large deflections. The first part of this section presents a method to retrieve the deformed configuration of the needle from a scanner image. Thus, the convected coordinates of needles inserted into tissue are retrieved from the CT scans of the experimental needle insertions into pig shoulder presented in Section 2.1 (Fig. 6a) and then used as reference needles. Finally, in the second part of this section, the needle sensor positioning is defined as a minimization problem and its resolution is addressed.

\subsubsection{Reconstruction of Needle Deformed Configuration}

This section describes the method to recover the 3D convected coordinates of a needle from a scanner image. This method use the hypothesis and the deformation model presented in Section 4.1.2.

Needle Voxel Segmentation The CT scan is segmented in order to separate needle voxels from surrounding tissues. The seeded region growing technique is particularly appropriate because of the difference in voxel density between the tissues and the needle [2]. This algorithm was implemented as a plug-in in the medical visualization software CamiTK [11]. The results of the segmentation is a set containing all the needle voxels, as shown in Fig. 6 b.

Needle Shape Smoothing The needle voxels are the result of a noisy spatial discretization of the needle shape. Therefore, it is possible to approximate the needle shape by smoothing the needle voxels. The smoothing method used in this paper is the B-spline smoothing, one of the most commonly used method to smooth data, which consists in performing a regularized regression on a $\mathrm{B}$-spline basis.

B-splines are piecewise polynomials defined by their degrees, knots and control points [6]. The degree of the spline is denoted $n$, the number of knots is denoted $n_{k}$ and the number of control points is denoted $n_{c}$. The knots are denoted $\left(t_{i}\right)_{i=1, \ldots, n_{k}} \in \mathbb{R}^{n_{k}}$ and the control points are denoted 


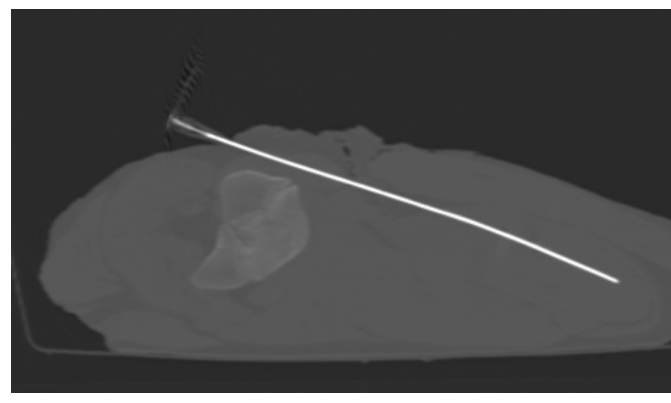

(a)

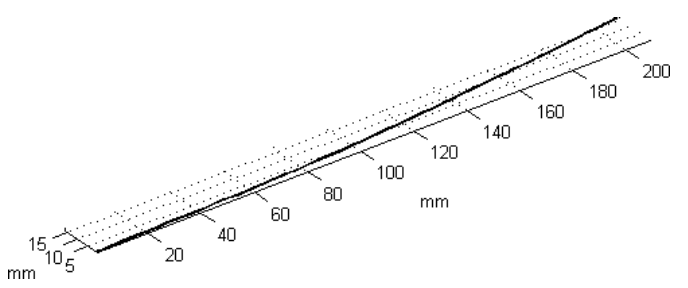

(c)

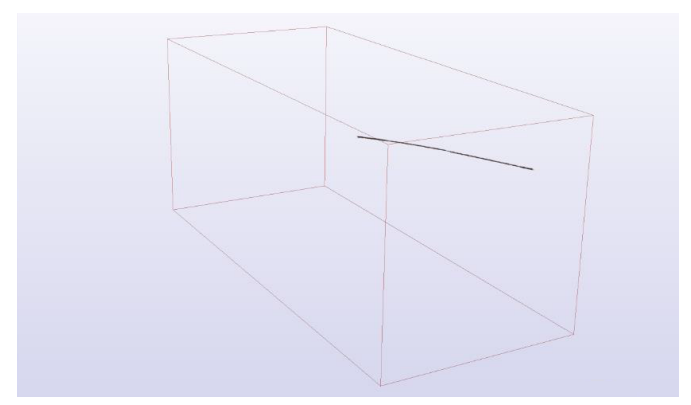

(b)

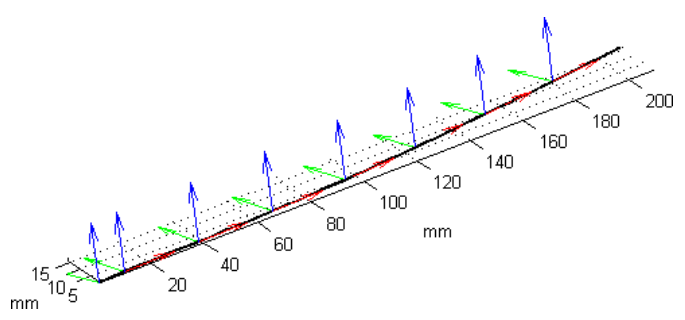

(d)

Fig. 6 Presentation of the different stages of the reconstruction process of a deformed needle. (a) The needle selected for illustration purpose has a planar deformation so that its insertion is fully visible in one slice of the CT scan. (b) The needle voxels are extracted from the CT scans by segmentation (Section 4.2.1). (c) The shape of the needle as a 3D B-spline is obtained by spline smoothing (Section 4.2.1). (d) The convected coordinates of the needle are computed from its shape (Section 4.2.1)

$\left(\mathbf{P}_{i}\right)_{i=1, \ldots, n_{c}}$ with $\forall i, \mathbf{P}_{i} \in \mathbb{R}^{3}$. Let $\mathbf{S}$ be the B-spline which is defined by:

$\mathbf{S}(t)=\sum_{i=1}^{n_{c}} B_{i, n}(t) \mathbf{P}_{i}$

with the following recursive definition of the B-spline basis $\operatorname{set}\left(B_{i, n}\right)_{i=1, . ., n_{c}}$ :

$B_{i, 0}(t)= \begin{cases}1 & \text { if } t_{i} \leq t \leq t_{i+1} \\ 0 & \text { otherwise }\end{cases}$

$B_{i, n}(t)=\frac{t-t_{i}}{t_{i+n}-t_{i}} B_{i, n-1}(t)+\frac{t_{i+n+1}-t}{t_{i+n+1}-t_{i+1}} B_{i+1, n-1}(t)$

The definition of the B-spline basis ensures that the B-spline $\mathbf{S}$ is of class $C^{n-1}$. This property justifies the use of B-splines here as it ensures that the resulting approximation of the needle shape will be smooth.

Let $\left(\mathbf{X}_{i}\right)_{i=1, \ldots, n_{d}}$ be the set of the $n_{d}$ data points to smooth, here the needle voxels, with $\mathbf{X}_{k} \in \mathbb{R}^{3}$ containing the $3 \mathrm{D}$ coordinates of the $k^{\text {th }}$ voxel. Voxels have been pre-ordered using the PCA-based method proposed by Furferi et al [38]. The data points are associated to the B-spline points through the parameters $\left(\bar{t}_{i}\right)_{i=1, . ., n_{d}}$ which reflect the distribution of the data points along the B-spline. These parameters were obtained using the centripetal parameterization method proposed by Lee [23] which is one of the most common method used in solving the parameterization problem [14]. The weight $w_{k}$ represents the contribution assigned to the data point $\mathbf{X}_{k}$ during the smoothing. The voxel values were used as weights because they decrease with distance between the voxel and the needle neutral axis, therefore giving less significancy to voxels located far from the needle neutral axis. The smoothing system takes the following form:

$$
\sum_{l=1}^{n_{d}} w_{l}\left\|\mathbf{S}\left(\bar{t}_{l}\right)-\mathbf{X}_{l}\right\|^{2}+\lambda \int_{0}^{1}\left\|\mathbf{S}^{(m)}(t)\right\|^{2} d t
$$

with $m$ the degree of smoothing and $\mathbf{S}$ a B-spline of degree $2 m-1$ [10]. The value of the smoothing degree for (43) is set to $m=3$ which corresponds to quintic B-splines and allows correct estimates of first and second derivatives of the data to be obtained [49]. The quality of these estimates is crucial as they are further used to compute curvature and bending angle functions. The smoothing B-spline estimate is defined to be the minimization of (43) over the control points $\left(\mathbf{P}_{i}\right)_{i=1, \ldots, n_{c}}$. The first term of (43) reflects the accuracy of the data points whereas the second term expresses the roughness of the estimate. The trade-off between these two terms is the variable $\lambda$, called the smoothing parameter.

The choice of the smoothing parameter $\lambda$ is critical as it impacts the accuracy and the roughness of the solution. The optimal value of $\lambda$ can be defined as the value which minimizes the shape error between the deformed needle and the reconstruction from its voxels discretization. This value is shape dependant and then each deformed needle has its 
own optimal value of $\lambda$. In our case, the deformed needle data is unavailable as we only have the discrete voxels from segmented scans. Nevertheless, it is possible to overcome this situation by using methods such as Generalized Cross-Validation [10] which gives us the optimal value of the smoothing parameter for a given deformed needle from its voxel discretization. Finally the shape of the needle in the CT scan is reconstructed, as shown in Fig. 6c.

Computation of the Needle Convected Coordinates The simplest way to obtain the convected coordinates of the needle consists of rotating the Serret-Frenet frame as shown in (27). Nevertheless, as the computation of the SerretFrenet frame can result in an undesirable rotation around the tangent of the curve, especially when the curve is almost straight, more stable computations of other frames are often preferred to the Serret-Frenet frame [47]. The method employed here is the Double reflection method, proposed by Wang et al., which computes the rotation-minimizing frames (RMF) of a curve with a fourth order of approximation error. The RMF is a 3D orthonormal frame based on Bishop application of parallel transport to curve framing composed of a vector tangent to the curve and two vectors whose derivatives are tangential [3]. Equation (29) and (30) shows that when the torsion of the needle is ignored the derivatives $\mathbf{N}_{\mathbf{1}}{ }^{\prime}=-\kappa \cos \theta \mathbf{T}$ and $\mathbf{N}_{\mathbf{2}}{ }^{\prime}=\kappa \sin \theta \mathbf{T}$ are tangential and then the convected material frame of the needle $\left(\mathbf{T}, \mathbf{N}_{\mathbf{1}}, \mathbf{N}_{\mathbf{2}}\right)$ is a RMF which can be computed with the double reflection method, as shown in Fig. 6d.

\subsubsection{Optimization Problem}

The set containing the convected coordinates of the reference needles is denoted $\mathscr{N}$, it was obtained from the pig needle insertion experimental data presented in Section 2.1 which was processed as described in Section 4.2.1. The convected coordinates of a needle can then be used to reconstruct the needle shape and to simulate strain sensor measures. The error measure used is the tip error employed by Park et al. [31] and Seifabadi et al. [42], which is the distance between the tip of the reference needle and the tip of its reconstructed shape. This choice is driven by the fact that a correctly positioned needle tip is one of the principal factors for success in interventional radiology procedures. The function giving the tip position of a needle is denoted tip. The optimal sensor locations are the locations that minimize the sum of squared error measure between the reference needle $N$ and its reconstructed shape $\operatorname{rec}(N, \mathbf{L})$ from given sensor locations $\mathbf{L}$. Let $\mathscr{L}$ be the set of locations of $n_{t}$ sensor triplets on a needle of length $L$. The problem of the optimal sensor triplet locations is then defined as the following optimization problem:

$\underset{\mathbf{L} \in \mathscr{L}}{\operatorname{minimize}} \sum_{N \in \mathscr{N}}\|\operatorname{tip}(N)-\operatorname{tip}(\operatorname{rec}(N, \mathbf{L}))\|^{2}$

This optimization problem belongs to the Mixed Integer Nonlinear Programming (MINLP) family as it is a problem with a nonlinear objective function and the solutions considered are integers. Thus MINLP optimization algorithms have been used to solve (44). The algorithms selected are NOMAD[22], KNITRO[8] and MATLAB genetic algorithm. Finally, the locations minimizing (44) are the optimal sensor triplet locations.

\section{Results}

The works concerning instrumented needles differ by the number of triplets per needle. This number is the number of cross section instrumented with strain sensors, mostly based on fiber Bragg gratings technology. The instrumentation presented in the litterature use two [31], three [15, 42], four [1, 39] or five [17] triplets. Consequently, in order to cover all possible cases, the number of triplet considered is comprised between one (the minimum number of triplet required to instrument a needle) and five (the maximum number of triplet of an instrumented needle presented in the litterature). The minimization described in Section 4.2.2 was then performed on the set of the experimental needle insertions for a number of sensor triplets from one to five. The resulting optimal locations of sensor triplets are presented in Table 2. Locations are specified in $\mathrm{mm}$ from the proximal extremity of the needle.

Table 2 Optimal sensor triplet locations. Locations are expressed as distances in $\mathrm{mm}$ from the proximal extremity of the needle.

\begin{tabular}{|c|c|c|c|c|c|}
\hline \multirow{2}{*}{$\begin{array}{c}\text { Number of } \\
\text { sensors triplets }\end{array}$} & \multicolumn{6}{|c|}{ Optimal sensor triplet locations (mm) } \\
\cline { 2 - 6 } & $l_{1}$ & $l_{2}$ & $l_{3}$ & $l_{4}$ & $l_{5}$ \\
\hline 1 & 81 & & & & \\
\hline 2 & 28 & 101 & & & \\
\hline 3 & 25 & 77 & 136 & & \\
\hline 4 & 9 & 40 & 95 & 144 & \\
\hline 5 & 11 & 34 & 77 & 128 & 171 \\
\hline
\end{tabular}

For comparison purposes, optimal locations were used along with equidistant locations to reconstruct the experimental and clinical needle sets. Boxplots of tip errors of the reconstructed needles are presented in Fig. 7. These results show that, for both needle sets, the quartiles and the maximal tip error are smaller with sensors placed at optimal locations than with sensors placed at equidistant locations. The mean tip error for both sets, presented in Table 3 , is also 
Table 3 Reconstruction results of the needle from experimental and clinical insertions with sensors triplets placed at equidistant and optimal locations. P-values were calculated using a Wilcoxon signed-rank test on tip errors.

\begin{tabular}{|c|c|c|c|c|c|c|c|c|}
\hline \multirow{3}{*}{ Needle set } & \multirow{3}{*}{$\begin{array}{c}\text { Number of } \\
\text { sensors triplets }\end{array}$} & \multicolumn{4}{|c|}{ Reconstruction tip error } & \multirow{2}{*}{\multicolumn{3}{|c|}{ Gain in accuracy }} \\
\hline & & \multicolumn{2}{|c|}{ Equidistant locations } & \multicolumn{2}{|c|}{ Optimal locations } & & & \\
\hline & & Mean $(\mathrm{mm})$ & Median (mm) & Mean $(\mathrm{mm})$ & Median (mm) & Mean \pm SD (mm) & Relative mean $(\%)$ & $\mathrm{P}$-value \\
\hline \multirow{5}{*}{ Experimental } & 1 & 9.67 & 8.43 & 8.03 & 8.08 & $1.64 \pm 5.58$ & $17 \%$ & 0.053 \\
\hline & 2 & 5.57 & 5.12 & 3.93 & 3.69 & $1.64 \pm 3.29$ & $29 \%$ & 0.002 \\
\hline & 3 & 4.32 & 3.45 & 3.25 & 2.67 & $1.07 \pm 3.04$ & $25 \%$ & 0.017 \\
\hline & 4 & 3.60 & 3.08 & 2.65 & 2.04 & $0.95 \pm 2.51$ & $26 \%$ & 0.010 \\
\hline & 5 & 3.38 & 2.65 & 2.21 & 1.89 & $1.17 \pm 1.87$ & $35 \%$ & $<0.001$ \\
\hline \multirow{5}{*}{ Clinical } & 1 & 8.08 & 7.13 & 8.04 & 6.45 & $0.04 \pm 2.21$ & $<1 \%$ & 1.000 \\
\hline & 2 & 4.25 & 3.86 & 2.33 & 2.40 & $1.92 \pm 2.56$ & $45 \%$ & 0.063 \\
\hline & 3 & 2.86 & 2.75 & 1.41 & 1.18 & $1.45 \pm 1.28$ & $51 \%$ & 0.063 \\
\hline & 4 & 1.71 & 1.00 & 0.91 & 0.64 & $0.80 \pm 0.76$ & $47 \%$ & 0.031 \\
\hline & 5 & 1.62 & 0.95 & 0.91 & 0.58 & $0.70 \pm 1.04$ & $43 \%$ & 0.031 \\
\hline
\end{tabular}

smaller with optimal locations. Therefore, the gain of accuracy which is defined by the difference between the tip error with equidistant locations and tip error with optimal locations is positive in every case. The gain of accuracy is comprised between $0.95 \mathrm{~mm}$ and $1.64 \mathrm{~mm}$ for experimental needle insertions and between $0.04 \mathrm{~mm}$ and $1.92 \mathrm{~mm}$ for clinical needle insertions. To determine the statistical significance of these gains, a Wilcoxon signed-rank test was performed on the tip errors to test whether the mean tip error differs. The significance threshold was set at 0.05 . The resulting p-values, presented in Table 3 , show that there is significant differences between the tip errors of reconstructions of the experimental needle insertions with a number of triplets greater than 2 and between the tip errors of reconstructions of the clinical needle insertions with a number of triplets greater than 4. Finally, when the gain in accuracy is statistically significant, the use of optimal locations improves in average the reconstruction accuracy by $25 \%$ to $35 \%$ for experimental needle insertions, as presented in Table 3 , and by $43 \%$ to $47 \%$ for clinical needle insertions, as shown in Table 3.

Needles have also been reconstructed using optimal sensors triplets locations coming from the works of Park et al. [31] and Seifabadi et al. [42]. The locations provided in Park et al. [31] for two sensor triplets on a $150 \mathrm{~mm}$ needle corresponds to $15 \%$ and $57 \%$ of the needle length and locations provided in Seifabadi et al. [42] for three sensor triplets on a $110 \mathrm{~mm}$ needle corresponds to $13 \%, 39 \%$ and $68 \%$ of the needle length. Reconstruction results of both needle insertions sets using those values as sensors triplets locations are presented in Table 4 and Table 5. Results show there is a statistically significant gain in accuracy using the optimal locations instead of the locations obtained from Park et al. [31] for the reconstruction of the experimental needle set and that the gain is also statistically significant when the optimal locations are used instead of locations obtained from Seifabadi et al. [42] for the reconstruction of both experimental and clinical needle sets. The use of optimal locations thus improves the reconstruction accuracy by $11 \%$ compared to locations from Park et al. [31] and between 62\% and 69\% compared to locations from Seifabadi et al. [42].

\section{Discussion}

The locations of the strain sensors on the needle are critical because of their impact on the needle shape reconstruction. Therefore, as the number of embedded sensors on needles is limited due to technical restrictions, positioning sensors appropriately constitutes an efficient way to improve reconstruction accuracy. A new method to compute the optimal locations of the strain sensor triplets of a needle has been proposed in this study. The results demonstrate that the reconstruction is more accurate with these locations compared to equidistant locations and locations presented in the literature.

The optimal locations computed using experimental needle insertions data and presented in Table 2 demonstrate that optimal locations are much closer to the proximal extremity than the distal extremity of the needle. In fact, the closer from the proximal extremity a reconstruction imprecision is made, the worst the reconstruction is. Hence the need to acquire more deformation informations near the proximal extremity, which explains a larger sensor density near that area. The results of reconstruction with equidistant and optimal locations show that in every case the tip error is smaller when the sensor triplets are located at optimal locations. For a number of triplets greater than two, the gain in accuracy stays similar in each set $(29 \%, 25 \%, 26 \%, 35 \%$ for experimental needle set and $45 \%, 51 \%, 47 \%, 53 \%$ for clinical needle set) and do not have specific trends. On the opposite, when the number of triplets increases the standard deviation decreases, meaning that the dispersion of the gain in accu- 
Table 4 Reconstruction results of the needle from experimental and clinical insertions with sensors triplets placed at locations obtained from Park et al. [31] and optimal locations. P-values were calculated using a Wilcoxon signed-rank test on tip errors.

\begin{tabular}{|c|c|c|c|c|c|c|c|c|}
\hline \multirow{2}{*}{ Needle set } & \multirow{2}{*}{$\begin{array}{c}\text { Number of } \\
\text { sensors triplets }\end{array}$} & \multicolumn{3}{|c|}{ Reconstruction tip error } & \multicolumn{3}{c|}{ Gain in accuracy } \\
\cline { 3 - 9 } & & Locations from Park et al. & Optimal locations & \multicolumn{3}{c|}{} \\
\cline { 3 - 9 } & & Mean (mm) & Median (mm) & Mean (mm) & Median (mm) & Mean \pm SD (mm) & Relative mean (\%) & P-value \\
\hline Experimental & 2 & 4.43 & 4.30 & 3.93 & 3.69 & $0.51 \pm 1.86$ & $11 \%$ & 0.045 \\
\hline Clinical & 2 & 2.58 & 2.55 & 2.33 & 2.40 & $0.25 \pm 0.99$ & $10 \%$ & 0.188 \\
\hline
\end{tabular}

Table 5 Reconstruction results of the needle from experimental and clinical insertions with sensors triplets placed at locations obtained from Seifabadi et al. [42]. P-values were calculated using a Wilcoxon signed-rank test on tip errors.

\begin{tabular}{|c|c|c|c|c|c|c|c|c|}
\hline \multirow{2}{*}{ Needle set } & \multirow{2}{*}{$\begin{array}{c}\text { Number of } \\
\text { sensors triplets }\end{array}$} & \multicolumn{3}{|c|}{ Reconstruction tip error } & \multicolumn{3}{c|}{ Gain in accuracy } \\
\cline { 3 - 9 } & & Locations from Seifabadi et al. & \multicolumn{2}{|c|}{ Optimal locations } & \multicolumn{3}{c|}{} \\
\cline { 3 - 9 } & & Mean $(\mathrm{mm})$ & Median $(\mathrm{mm})$ & Mean $(\mathrm{mm})$ & Median $(\mathrm{mm})$ & Mean \pm SD $(\mathrm{mm})$ & Relative mean $(\%)$ & P-value \\
\hline Experimental & 3 & 8.66 & 7.38 & 3.25 & 2.67 & $5.41 \pm 4.93$ & $62 \%$ & $<0.001$ \\
\hline Clinical & 3 & 4.52 & 4.42 & 1.41 & 1.18 & $3.11 \pm 3.37$ & $69 \%$ & 0.031 \\
\hline
\end{tabular}

racy decreases as well. In this case, it signifies there is more and more cases where the reconstruction is better with the optimal locations than with the equidistant locations. One explanation is that with the increase of the sensor density on the needle the deformations are less likely to be unnoticed. It thus minimizes the randomness of the deformations on the reconstruction accuracy. Consequently, as the p-values decreases, the gain in accuracy obtained with the use of the optimal locations are more and more statistically significant. For the experimental needle insertions the gain in accuracy becomes statistically significant when the number of triplets considered is greater than 2 and for the clinical needle insertions when the number of triplets is greater than 4 .

To the best of the author's knowledge there is no work in the literature concerning optimal locations of sensor triplets for a $200 \mathrm{~mm}$ long needle. Park et al. [31] presented optimal locations for a $150 \mathrm{~mm}$ long needle and Seifabadi et al. [42] for a $110 \mathrm{~mm}$ long needle. The locations were computed from uniformly distributed load and concentrated forces at the extremity. The chosen load values are reflecting the interaction with the tissue during the insertion and are thus independant from the needle length. Optimal locations from Park et al. [31] and Seifabadi et al. [42] have then been scaled to fit on $200 \mathrm{~mm}$ needles, giving optimal locations of $29 \mathrm{~mm}$ and $113 \mathrm{~mm}$ for Park et al. [31] and $55 \mathrm{~mm}, 144 \mathrm{~mm}$ and $180 \mathrm{~mm}$ for Seifabadi et al. [42]. The optimal locations for two triplets presented in Table 3 are very close to those obtained from Park et al. [31] whereas the optimal locations for three triplets are very distant from the one obtained from Seifabadi et al. [42]. One of the reason is that, in the work of Seifabadi, the number of sensors activated varies during the insertion. Nevertheless, when the needle is fully inserted all sensors are activated, as in our case, which makes the use of these locations relevant. In every case the tip error of the re- construction are smaller with the optimal locations than with locations obtained from the literature. The gain in accuracy using the optimal locations is $10 \%$ and $11 \%$ compared to Park et al. [31] and 62\% and 69\% compared to Seifabadi et al. [42].

These gains in accuracy are statistically significant except for the reconstruction of the clinical needle set with two triplets. This result is due to the limited number of elements of the clinical needle set. This phenomenon, associated with the effect discussed above about reconstruction with a limited number of triplet, is also the cause of the almost null gain in accuracy $(0.04 \%)$ when reconstructing with one triplet the clinical needle set using optimal locations compared to equidistant locations. Thus, the reduced size of the clinical needle set constitutes a limitation of the results presented here and could be corrected by considering a larger set of needle insertions. Another limitation of our method is the quality of approximation of the needle in the scan which has an impact on the approximation of the curvature and bending angle functions and then on the results of the optimal sensor locations. This limit is present regardless of the approximation method used. An interesting development would be to characterize the approximation error. Many articles deal with the approximation error of a smoothing spline $[33,36]$ and their work would constitute a first step towards evaluating the quality of the approximation of the needle shape.

Finally the reconstructions of the experimental and clinical needle sets validate the proposed approach as the optimal locations provide improvement of the reconstruction accuracy. In particular, the gain in accuracy obtained with the reconstructions of the clinical needle set using the same type of needles demonstrate the potential of the optimal locations for clinical application. We underline the fact that 

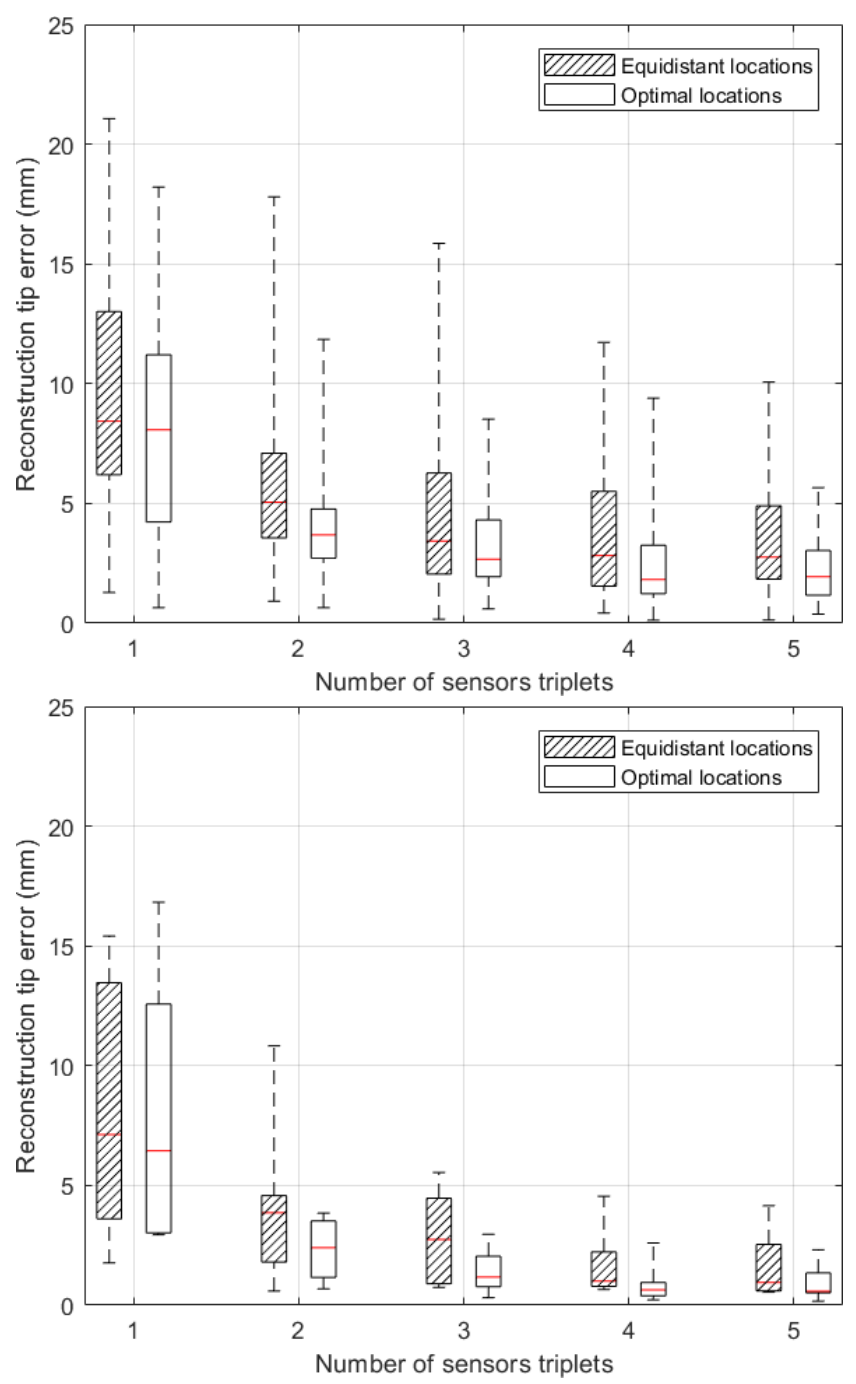

Fig. 7 Boxplots of the reconstruction tip errors of the experimental needle insertions (top) and clinical needle insertions (bottom) according to the sensor triplets locations.

the optimal locations, determined from a set of needle insertions in pig tissue, improve the reconstruction of a set of needle inserted in real human tissue. This suggests that the optimal locations are not limited to the type of tissues with which they were computed. Consequently, these results support the use of optimal locations given by our method for positioning the strain sensors on an instrumented needle. The non-specificity of the method to characteristics such as tissue and the insertions are strong indications that the method would work with other type of soft tissues or insertions. To this end, the similarity of the tissues of both dataset and the exhaustivity of insertions performed experimentally seem to be key factors of the method success.

\section{Conclusion}

Finally, in this paper, we presented a 3D large deflection needle shape reconstruction method and an experimentallybased method for positioning strain sensors on needles. The optimal sensor locations were computed from experimental data of needle insertions into pig tissue. Reconstruction simulations from clinical data of needle insertions using the same type of needles showed that using the optimal locations instead of equidistant locations improves the needle shape reconstruction in average. We conclude that the needle reconstruction accuracy benefit from this new sensor positioning method and its use on instrumented needles in navigation systems would result in a better location of the needle in the tissues, improving the therapeutic result of the procedure. Currently most instrumented needle prototypes use fiber Bragg gratings as strain sensors technology. These sensors consist in reflectors implemented in optical fibers embedded at the surface of the needle. As these sensors are expensive, the number of triplets of the needle has thus a significant impact on the price of the needle instrumentation. Consequently, the method presented in this paper can provide positive effects on the price-accuracy trade-off, either by improving the accuracy for a given number of sensors or reducing the number of sensors necessary for a given accuracy. Prototypes of instrumented needles are currently being developed by Bonvilain et al. with successfull results concerning instrumentation of needles with strain gauges $[4,5]$. Experimental insertions of needles with strain gauges will then be realised as future work when a working prototype of instrumented needle will be available. It will then be possible to compare reconstruction results from different sensor locations and to evaluate the gain of accuracy obtained with optimal locations directly from experimentation.

\section{Conflicts of interest}

The authors declare they have no conflict of interests.

\section{Acknowledgment}

This work is part of the project GAME-D, financed by the French National Agency for Research (ref: ANR-12-TECS0019) and supported by Laboratory of Excellence CAMI (ref: ANR-11-LABX-0004-01).

The authors would like to thank Benjamin Spencer and Cecilia Hughes for their English reviews and corrections.

\section{References}

1. Abayazid, M., Kemp, M., Misra, S.: 3d flexible needle steering in soft-tissue phantoms using fiber bragg grat- 
ing sensors. In: Proc. IEEE International Conference on Robotics and Automation (ICRA), pp. 5843-5849 (2013). DOI 10.1109/ICRA.2013.6631418

2. Adams, R., Bischof, L.: Seeded region growing. IEEE Transactions on pattern analysis and machine intelligence 16(6), 641-647 (1994)

3. Bishop, R.L.: There is more than one way to frame a curve. The American Mathematical Monthly 82(3), 246-251 (1975). URL http://www.jstor.org/stable/2319846

4. Bonvilain, A., Gangneron, M.: Characterization of strain microgauges for the monitoring of the deformations of a medical needle during its insertion in human tissues. Microsystem Technologies 22(3), 551556 (2016). DOI 10.1007/s00542-015-2588-2. URL https://doi.org/10.1007/s00542-015-2588-2

5. Bonvilain, A., Zanardelli, L., Carriquiry, A.: Piezoresistif microsensors for an instrumented medical needle for its real time monitoring in a microlocalization tool. Microsystem Technologies 24(7), 31613167 (2018). DOI 10.1007/s00542-018-3814-5. URL https://doi.org/10.1007/s00542-018-3814-5

6. de Boor, C.: A Practical Guide to Splines. SpringerVerlag New York (1978)

7. Brett, P.N., Parker, T., Harrison, A.J., Thomas, T.A., Carr, A.: Simulation of resistance forces acting on surgical needles. Proceedings of the Institution of Mechanical Engineers, Part H: Journal of Engineering in Medicine 211(4), 335-347 (1997)

8. Byrd, R.H., Nocedal, J., Waltz, R.A.: Knitro: An integrated package for nonlinear optimization. In: Large-scale nonlinear optimization, pp. 35-59. Springer (2006)

9. Cosserat, E., Cosserat, F., et al.: Théorie des corps déformables (1909)

10. Craven, P., Wahba, G.: Smoothing noisy data with spline functions. Numerische Mathematik 31(4), 377-403 (1978). DOI 10.1007/BF01404567. URL http://dx.doi.org/10.1007/BF01404567

11. Fouard, C., Deram, A., Keraval, Y., Promayon, E.: CamiTK: a modular framework integrating visualization, image processing and biomechanical modeling. In: Y. Payan (ed.) Soft Tissue Biomechanical Modeling for Computer Assisted Surgery, pp. 323-354 (2012)

12. Guggenheimer, H.: Computing frames along a trajectory. Computer Aided Geometric Design 6(1), 77 - 78 (1989)

13. Hairer, E., Wanner, G., Lubich, C.: Geometric Numerical Integration. Structure-Preserving Algorithms for Ordinary Differential Equations, 2 edn. (2006)

14. Haron, H., Rehman, A., Adi, D., Lim, S., Saba, T.: Parameterization method on b-spline curve. Mathematical Problems in Engineering 2012 (2012)
15. van der Heiden, M.S., Henken, K.R., Chen, L.K., van den Bosch, B.G., van den Braber, R., Dankelman, J., van den Dobbelsteen, J.: Accurate and efficient fiber optical shape sensor for mri compatible minimally invasive instruments (2012). DOI 10.1117/12.981141. URL http://dx.doi.org/10.1117/12.981141

16. Henken, K., Gerwen, D.V., Dankelman, J., Dobbelsteen, J.V.D.: Accuracy of needle position measurements using fiber bragg gratings. Minimally Invasive Therapy \& Allied Technologies 21(6), 408-414 (2012). DOI 10.3109/13645706.2012.666251

17. Henken, K.R., Dankelman, J., van den Dobbelsteen, J.J., Cheng, L.K., van der Heiden, M.S.: Error analysis of fbg-based shape sensors for medical needle tracking 19(5), 1523-1531 (2014). DOI 10.1109/TMECH.2013.2287764

18. Hocking, G., Hebard, S., Mitchell, C.H.: A review of the benefits and pitfalls of phantoms in ultrasoundguided regional anesthesia. Regional anesthesia and pain medicine 36(2), 162-170 (2011)

19. Jiang, S., Li, P., Yu, Y., Liu, J., Yang, Z.: Experimental study of needletissue interaction forces: Effect of needle geometries, insertion methods and tissue characteristics. Journal of Biomechanics 47(13), 3344 - 3353 (2014). DOI https://doi.org/10.1016/j.jbiomech.2014.08.007

20. Kim, B., Ha, J., Park, F.C., Dupont, P.E.: Optimizing curvature sensor placement for fast, accurate shape sensing of continuum robots. In: 2014 IEEE International Conference on Robotics and Automation (ICRA), pp. 5374-5379 (2014). DOI 10.1109/ICRA.2014.6907649

21. Kirchhoff, G.: Uber das gleichgewicht und die bewegung eines unendlich dunnen elastischen stabes. J. Reine Angew. Math. 56, 285-313 (1859)

22. Le Digabel, S.: Algorithm 909: Nomad: Nonlinear optimization with the mads algorithm. ACM Trans. Math. Softw. 37(4), 44:1-44:15 (2011). DOI 10.1145/1916461.1916468. URL http://doi.acm.org/10.1145/1916461.1916468

23. Lee, E.: Choosing nodes in parametric curve interpolation. Computer-Aided Design 21(6), 363 - 370 (1989). DOI https://doi.org/10.1016/0010-4485(89)90003-1

24. Love, A.E.H.: A treatise on the mathematical theory of elasticity, 2 edn. (1906)

25. Magnus, W.: On the exponential solution of differential equations for a linear operator. Communications on Pure and Applied Mathematics 7(4), 649673 (1954). DOI 10.1002/cpa.3160070404. URL http://dx.doi.org/10.1002/cpa.3160070404

26. Mahoney, A.W., Bruns, T.L., Swaney, P.J., Webster, R.J.: On the inseparable nature of sensor selection, sensor placement, and state estimation for continuum 
robots or where to put your sensors and how to use them. In: Robotics and Automation (ICRA), 2016 IEEE International Conference on, pp. 4472-4478. IEEE (2016)

27. Monasa, F., Lewis, G.: Large deflections of point loaded cantilevers with nonlinear behaviour. ZAMP Zeitschrift fr angewandte Mathematik und Physik 34(1), 124-130 (1983). DOI 10.1007/bf00962621

28. Moon, H., Jeong, J., Kang, S., Kim, K., Song, Y.W., Kim, J.: Fiber-bragg-grating-based ultrathin shape sensors displaying single-channel sweeping for minimally invasive surgery. Optics and Lasers in Engineering 59, 50 - 55 (2014). DOI http://dx.doi.org/10.1016/j.optlaseng.2014.03.005

29. Moreira, P., Misra, S.: Biomechanics-based curvature estimation for ultrasound-guided flexible needle steering in biological tissues. Annals of Biomedical Engineering 43(8), 1716-1726 (2015). DOI 10.1007/s10439-014-1203-5. URL https://doi.org/10.1007/s10439-014-1203-5

30. Ng, K.W., Goh, J.Q., Foo, S.L., Ting, P.H., Lee, T.K.: Needle insertion forces studies for optimal surgical modeling. International Journal of Bioscience, Biochemistry and Bioinformatics 3(3), 187 (2013)

31. Park, Y.L., Elayaperumal, S., Daniel, B., Ryu, S.C., Shin, M., Savall, J., Black, R., Moslehi, B., Cutkosky, M.: Real-time estimation of 3-d needle shape and deflection for mri-guided interventions 15(6), 906-915 (2010). DOI 10.1109/TMECH.2010.2080360

32. Podder, T.K., Clark, D.P., Sherman, J.: Effects of tip geometry of surgical needle an assessment of force and deflection. Third European medical and biological engineering conference, Prague, Czech Republic pp. 16411644 (2005)

33. Ragozin, D.L.: Error bounds for derivative estimates based on spline smoothing of exact or noisy data. Journal of Approximation Theory 37(4), 335 - 355 (1983). DOI http://dx.doi.org/10.1016/0021-9045(83)90042-4

34. Rao, C.K., Deshpande, A.P.: Modelling of engineering materials. Ane Books Pvt Ltd (2010)

35. Reissner, E.: On one-dimensional large-displacement finite-strain beam theory. Studies in Applied Mathematics 52(2), 87-95 (1973)

36. Rice, J., Rosenblatt, M.: Integrated mean squared error of a smoothing spline. Journal of Approximation Theory 33(4), 353 - 369 (1981). DOI http://dx.doi.org/10.1016/0021-9045(81)90066-6

37. Robert, A.L., Chagnon, G., Bricault, I., Cinquin, P., Moreau-Gaudry, A.: A generic three-dimensional static force distribution basis for a medical needle inserted into soft tissue. Journal of the Mechanical Behavior of Biomedical Materials 28, 156 - 170 (2013). DOI http://dx.doi.org/10.1016/j.jmbbm.2013.07.023
38. Rocco Furferi Lapo Governi, M.P.Y.V.: From unordered point cloud to weighted b-spline - a novel pca-based method -. Applications of Mathematics and Computer Engineering (2011)

39. Roesthuis, R., Kemp, M., van den Dobbelsteen, J., Misra, S.: Three-dimensional needle shape reconstruction using an array of fiber bragg grating sensors 19(4), 1115-1126 (2014). DOI 10.1109/TMECH.2013.2269836

40. Roesthuis, R.J., Janssen, S., Misra, S.: On using an array of fiber bragg grating sensors for closed-loop control of flexible minimally invasive surgical instruments. In: 2013 IEEE/RSJ International Conference on Intelligent Robots and Systems, pp. 2545-2551 (2013). DOI 10.1109/IROS.2013.6696715

41. Rouchy, R., Moreau-Gaudry, A., Chipon, E., Aubry, S., Pazart, L., Lapuyade, B., Durand, M., Hajjam, M., Pottier, S., Renard, B., Logier, R., Orry, X., Cherifi, A., Quehen, E., Kervio, G., Favelle, O., Patat, F., De Kerviler, E., Hughes, C., Medici, M., Ghelfi, J., Mounier, A., Bricault, I.: Evaluation of the clinical benefit of an electromagnetic navigation system for ct-guided interventional radiology procedures in the thoraco-abdominal region compared with conventional ct guidance (ctnav ii): study protocol for a randomised controlled trial. Trials 18(1), 306 (2017). DOI 10.1186/s13063-017-2049-6. URL https://doi.org/10.1186/s13063-017-2049-6

42. Seifabadi, R., Gomez, E.E., Aalamifar, F., Fichtinger, G., Iordachita, I.: Real-time tracking of a bevel-tip needle with varying insertion depth: Toward teleoperated mri-guided needle steering. In: 2013 IEEE/RSJ International Conference on Intelligent Robots and Systems, pp. 469-476 (2013). DOI 10.1109/IROS.2013.6696393

43. Simo, J., Fox, D.: On a stress resultant geometrically exact shell model. part i: Formulation and optimal parametrization. Computer Methods in Applied Mechanics and Engineering 72(3), 267 - 304 (1989). DOI http://dx.doi.org/10.1016/0045-7825(89)90002-9

44. Sultan, S.F., Shorten, G., Iohom, G.: Simulators for training in ultrasound guided procedures. Medical ultrasonography 15(2), 125-131 (2013)

45. Todd, M.D., Stull, C.J., Dickerson, M.: A local material basis solution approach to reconstructing the threedimensional displacement of rod-like structures from strain measurements. Journal of Applied Mechanics 80(4) (2013)

46. Wan, G., Wei, Z., Gardi, L., Downey, D.B., Fenster, A.: Brachytherapy needle deflection evaluation and correction. Medical Physics 32(4), 902-909 (2005). DOI http://dx.doi.org/10.1118/1.1871372

47. Wang, W., Jüttler, B., Zheng, D., Liu, Y.: Computation of rotation minimizing frames. ACM Transactions on 
Graphics (TOG) 27(1), 2 (2008)

48. Whittaker, S., Lethbridge, G., Kim, C., Keon Cohen, Z., $\mathrm{Ng}$, I.: An ultrasound needle insertion guide in a porcine phantom model. Anaesthesia 68(8), 826-829. DOI 10.1111/anae. 12262

49. Wood, G.A.: Data smoothing and differentiation procedures in biomechanics. Exercise and sport sciences reviews 10(1), 308-362 (1982)

50. Xu, R., Yurkewich, A., Patel, R.V.: Shape sensing for torsionally compliant concentric-tube robots (2016). DOI 10.1117/12.2213128. URL http://dx.doi.org/10.1117/12.2213128

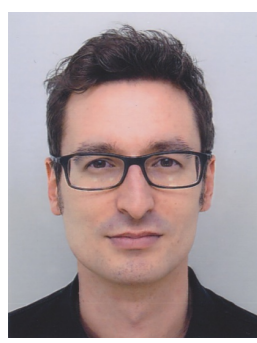

Pierre-Loup Schaefer received the M.Sc. degree in Computer Science and Applied Mathematics from ENSIMAG, Grenoble, France, in 2011. He received the Ph.D. degree in Applied Mathematics from Grenoble Alpes University in 2017 for his research in the field of computer assisted interventional radiology systems using instrumented needles.

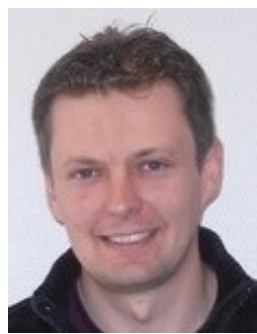

Grégory Chagnon engineer of Ecole Centrale de Nantes in 2000, received the M.Sc. degree in Mechanical Engineering from Nantes University, Grenoble, France, in 2000. He received the Ph.D. degree in Mechanical Engineering for his research in the field of mechanical analysis and modelling of rubber like materials in 2003. He is currently a member of the Biomedical and Mechanical engineering of Materials (BioMMat) team of the TIMCIMAG laboratory (Grenoble), and he is specialist in Mechanics of soft materials and also works on the development of smart biomedical devices.

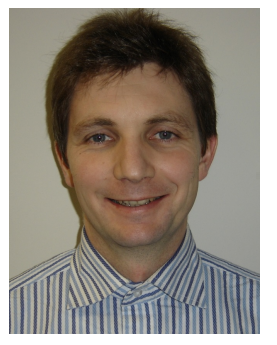

Alexandre Moreau-Gaudry engineer of ENSIMAG in 1996, received the M.Sc. degree in Applied Mathematics from Joseph Fourier University, Grenoble, France, in 1995. He received the Ph.D. degree in Applied Mathematics for his research in the field of Computer Assisted Medical Interventions in 2000 and received the degree in medicine in 2008 . He is a specialist in Medical Informatics at the Grenoble Alpes University Hospital, coordinating a research structure dedicated to the demonstration of the medical service associated to innovative medical device. His current research interests include the conception, development and evaluation of innovative medical devices with a specific focus on interventional radiology and orthopedics. 